\title{
The disparity of health facilities in an urban area discourages proposed treatment application in inoperable lung cancer patients
}

This article was published in the following Dove Press journal:

Cancer Management and Research

II November 2010

Number of times this article has been viewed

\section{Georgios Hillas' \\ Petros Bakakos ${ }^{2}$ \\ Miltiadis Trichas ${ }^{3}$ \\ Fotis Vlastos'}

'Department of Respiratory and Critical Care Medicine, "Sotiria" Chest

Diseases Hospital, Athens, Greece;

${ }^{2}$ Ist Respiratory Medicine

Department, University of Athens

Medical School, "Sotiria" Chest

Diseases Hospital, Athens, Greece;

${ }^{3}$ Radiotherapy Department,

Metropolitan Hospital, Neo Faliro,

Greece
Correspondence: Georgios Hillas

152 Mesogeion Avenue 115 27,

Athens, Greece

Tel +30210 7763566

Fax +302107473969

Email ghillas70@yahoo.gr
Objectives: Patients with a newly diagnosed non-small cell lung cancer (NSCLC) stage IIIB are offered chemoradiotherapy, as proposed by the current guidelines. This combination treatment is facilitated by the coexistence of corresponding departments in the same establishment. The geographical disparity of these health facilities influences patients' willingness to be treated and may influence their survival. This is an observational study that compares the survival of two groups of patients with NSCLC stage IIIB: those treated with chemoradiotherapy versus those treated only with chemotherapy. These two comparable groups were formed exclusively by patients' and/or their families' decisions.

Methods: One hundred fifteen consecutive NSCLC stage IIIB patients were included in the study. All were hospitalized in the biggest Chest Disease Hospital in Athens and were offered sequential chemoradiotherapy. Only 54 patients opted for the proposed treatment, while 61 decided to be treated with chemotherapy only, denying continuing their treatment in another health care unit (radiotherapy). Their survival and related factors were analyzed.

Results: Mean overall survival was estimated 10 months $(95 \%$ confidence interval $[\mathrm{CI}]$ : 7.96-12.04). Patients treated with chemoradiotherapy had almost double overall survival compared to those under chemotherapy $(P=0.001)$ : 13.6 months $(95 \%$ CI: $12.3-14.9)$ versus 7.5 (95\% CI: 6.1-8.9). Patients aged $\leq 65$ years $(P<0.001)$, smokers $(P<0.001)$, and those without a cancer history $(P<0.001)$ survived longer.

Conclusions: The lack of a radiotherapy department in a hospital providing chemotherapy impedes the application of current guidelines advocating combined radiochemotherapy. When recommended radiotherapy after six chemo cycles, half of the patients are unwilling to be displaced and do not follow the recommendations. This has an impact on patient survival.

Keywords: non-small cell lung cancer, survival, radiotherapy, chemotherapy, health facilities

\section{Introduction}

Lung cancer remains the leading cause of cancer deaths in both men and women, with approximately 1 to 2 million patients dying every year worldwide. ${ }^{1}$ Lung cancer causes more deaths than the next four most common cancers combined (colon, breast, pancreas, and prostate). ${ }^{2}$

Survival of patients with unresectable non-small cell lung cancer (NSCLC) stage IIIB remains dramatically poor. The treatment of advanced NSCLC includes two therapeutic options: chemo- and radiotherapy. Initial evidence suggested that combining radiotherapy with cisplatin alone or a cisplatin-based regimen modestly improves the median survival though the combined treatment compared with radiotherapy alone does not seem to improve the survival at 1 and 2 years. ${ }^{3}$ Later reports suggested that the 
addition of chemotherapy to radiotherapy improves survival in patients with locally advanced NSCLC. ${ }^{4}$

The use of concurrent chemo-radiotherapy in patients with advanced NSCLC was shown to be associated with a $14 \%$ reduction in death risk at 2 years compared to sequential chemoradiotherapy and with a $7 \%$ reduction of the same risk compared to radiotherapy alone. Nevertheless, toxicity concerns (mainly, acute oesophagitis and myelosuppression) did not permit the generalization of this type of therapeutic schedule. ${ }^{5}$ Theoretically, a sequential approach is mainly directed at eradication of micrometastatic disease, though concerns have been raised that the delay of radiotherapy resulting from the initial administration of chemotherapy allows for the accumulation of treatment-resistant clonogenic cells and decreases locoregional control. ${ }^{6}$ Combined modality therapy became the standard of care for NSCLC stage IIIB patients with good performance status.

In our hospital, which is the biggest chest disease hospital in Athens, patients with a newly diagnosed inoperable NSCLC stage IIIB and performance status 1 are offered the combined treatment proposed by the current guidelines. However, the concomitant version is not applicable due to the lack of a radiotherapy department. To receive the sequential version, patients have to displace to other hospitals across the Athens area. During the announcement of therapeutic schedule, patients and/or their families are informed that they could receive chemotherapy in the hosting hospital but that they have to displace to another hospital in order to receive radiotherapy. A number of patients are willing to opt for proposed displacement, while others opt for the least time-consuming treatment, namely chemotherapy, despite the existing scientific consensus that advocates the combined treatment. The patients decisions created two groups: patients treated with chemoradiotherapy versus those treated only with chemotherapy. The main end point of our study was to compare the survival of these two groups. Factors other than treatment modalities that were related to survival were also analyzed.

\section{Methods}

We conducted an observational study that recruited 115 consecutive patients with NSCLC stage IIIB between January 2002 and December 2008. Demographic variables, such as age, gender, and smoking habits were registered. Familial and personal cancer histories were also recorded. Tumor-related data included tumor histotypes and stage (TNM; WHO version 6). ${ }^{7}$

All patients received chemotherapy (a doublet of a platinum agent and docetaxel). The patients receiving the combined therapy were also treated with radical sequential radiotherapy. All patients completed six cycles of first-line chemotherapy. Fourteen patients of each group received second-line chemotherapy (vinorelbine plus gemcitabine) (Table 1). Patients and their families were informed, treated, and followed-up by the same medical staff, either in the hosting hospital or in the radiotherapy clinic.

Table I Patients' epidemiological and clinical characteristics

\begin{tabular}{|c|c|c|c|c|c|}
\hline \multirow[t]{2}{*}{ Main features } & & \multicolumn{2}{|c|}{$\begin{array}{l}\text { Chemoradiotherapy } \\
(n=54)\end{array}$} & \multicolumn{2}{|c|}{$\begin{array}{l}\text { Chemotherapy } \\
(n=6 I)\end{array}$} \\
\hline & & $n$ & Percentage \% & $\mathbf{n}$ & Percentage \% \\
\hline \multirow[t]{2}{*}{ Smoking } & Yes & 47 & 87 & 52 & 85.2 \\
\hline & No & 7 & 13 & 9 & 14.8 \\
\hline \multirow[t]{2}{*}{ Sex } & Male & 49 & 90.7 & 53 & 86.8 \\
\hline & Female & 5 & 9.3 & 8 & 13.2 \\
\hline \multirow[t]{2}{*}{ Age } & $\leq 65$ & 33 & 61.1 & 31 & 50.8 \\
\hline & $>65$ & 19 & 38.9 & 30 & 49.2 \\
\hline \multirow[t]{5}{*}{ Histological subtypes } & NSCLC & 54 & 46.9 & 61 & 53.1 \\
\hline & SQCLC & 22 & 40.7 & 22 & 36 \\
\hline & ADC & 17 & 31.5 & 23 & 37.7 \\
\hline & LCLC & 0 & 0 & 2 & 3.3 \\
\hline & NSCLCX & 15 & 27.8 & 14 & 23 \\
\hline \multirow[t]{2}{*}{ Cancer history } & Yes & 7 & 12.9 & 6 & 9.8 \\
\hline & No & 47 & 87.1 & 55 & 90.2 \\
\hline \multirow[t]{2}{*}{ COPD } & Yes & 19 & 35.2 & 23 & 37.7 \\
\hline & No & 35 & 64.8 & 38 & 62.3 \\
\hline Second-line & Yes & 14 & 26 & 14 & 23 \\
\hline chemotherapy & No & 40 & 74 & 51 & 77 \\
\hline
\end{tabular}

Abbreviations: ADC, adenocarcinoma; COPD, chronic obstructive pulmonary disease; LCLC, large cell lung cancer; NSCLC, non-small cell lung cancer; NSCLCx, undefined histological subtype of NSCLC. 
External beam radiotherapy was administered with 18 MV linear accelerator (SL20; Elekta, Norcross, GA) photons. Treatment fields encompassed the primary tumor and the involved lymph nodes with an appropriated safety margin. Computerized planning was utilized to deliver the required dose to the target volume while keeping the spinal cord dose below 45 Gy and V20 below 35\%. Continuouscourse treatment up to a total dose of $60 \mathrm{~Gy}$ in 30-33 daily fractions of 1.8-2 Gy (depending on the irradiated volume) was delivered over 6-7 weeks.

The course of the disease was followed. All patients and/or their families gave their written informed consent and the study was approved by the Ethics Committee of our hospital.

\section{Statistical analysis}

All analyses were performed using SPSS software (version 15.0; SPSS Inc., Chicago, IL). The Kaplan-Meier method was used to estimate survival in all groups. The survival times were not censored. Differences in the length of time to death were tested with the log-rank test (Mantel-Cox) if proportional hazard was identified as true or Breslow test (generalized Wilcoxon) when proportional hazard was identified as false. A secondary analysis was performed by using multivariate Cox regression analysis to estimate the hazard function. Statistical limits for removing and entering variables from the stepwise model were $P=0.10$ and $P=0.05$, respectively. All hazard ratios were estimated by the maximum likelihood estimator according to the Breslow method. Reported values were two-sided. The significant level for all tests was determined at $P=0.05$ (5\%).

\section{Results}

\section{Description of the cohort}

This study included 115 consecutive patients suffering from NSCLC stage IIIB. Their sex ratio was 102/13 (M/F). Their mean age $( \pm \mathrm{SD})$ was $64.45( \pm 6.7)$ years. The majority were smokers $(\mathrm{n}=99)$, with mean tobacco consumption 76 per/year. Their most frequent comorbidity was chronic obstructive pulmonary disease (COPD). Table 1 summarizes the main epidemiological and clinical characteristics of the two groups.

\section{Survival}

Acute radiation toxicity was minimal and there were no significant late toxicities. The mean overall survival of the cohort was estimated 10 months (95\% CI: 7.96-12.04) (Figure 1). Survival was correlated to histological subtypes: patients with squamous NSCLC presented a mean survival

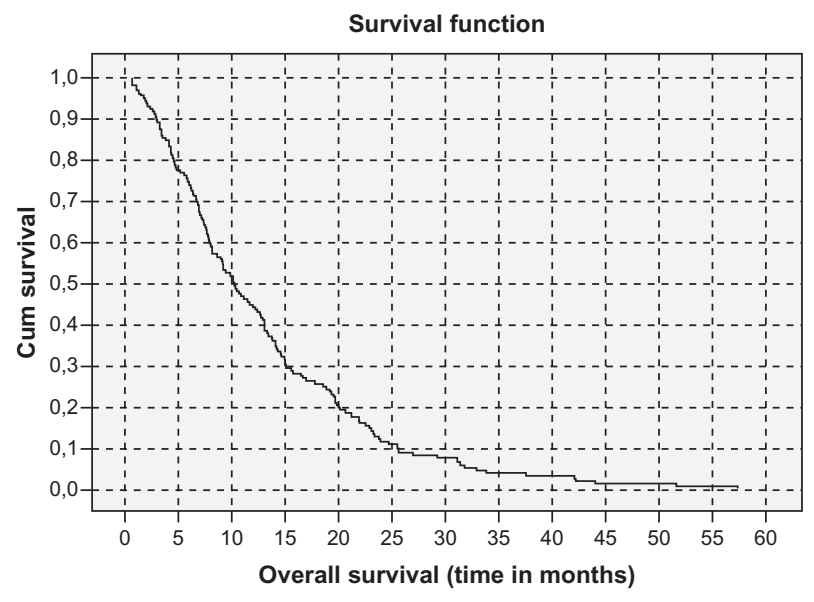

Figure I Overall survival of patients with non-small lung cancer stage IIIB.

of 10.7 months and those with ADC 10 months. These differences were significantly different in men (log-rank, Mantel-Cox: $P=0.002$; Breslow [generalized Wilcoxon]: $P=0.002)$ but not in women. Furthermore, these differences manifested among smokers (log-rank, Mantel-Cox: $P=0.003$; Breslow (generalized Wilcoxon): $P=0.002$ ) but not among nonsmokers.

Survival was significantly different between the two studied groups (log-rank test; $P=0.001$ ) in favor of the combined therapy arm (Figure 2). Patients receiving the combined treatment presented an almost double mean survival compared to those who received only chemotherapy (13.6 (12.3-14.9) versus 7.5 (6.1-8.9) months). This difference was manifest only among male patients $(P<0.001)$. Taking 65 years as a cutoff, we observed that the above-mentioned difference concerned only patients aged under 65 years $(P<0.001)$, while it subsided for patients aged above 65 years. The benefit of chemoradiotherapy is founded among smokers, while it was not demonstrated among nonsmokers.

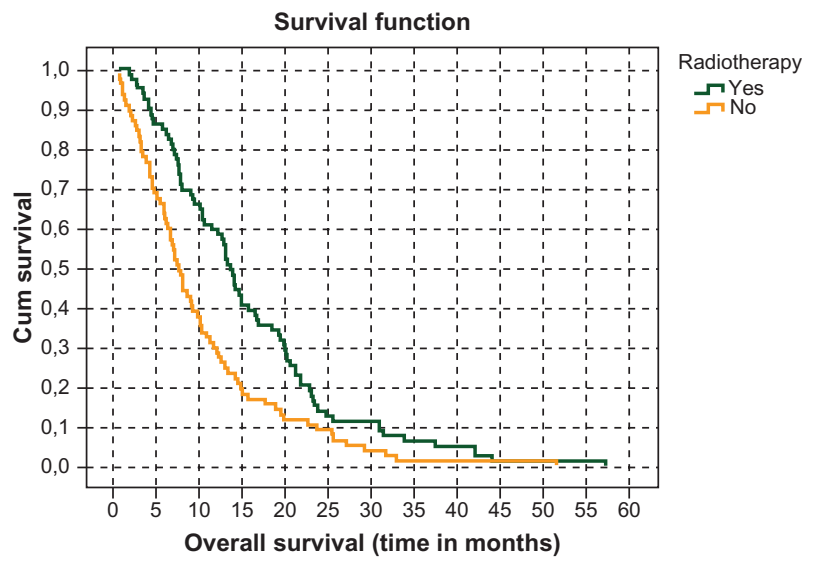

Figure 2 Overall survival of chemotherapy-treated lung cancer patients who received radiotherapy compared to those who didn't receive radiotherapy. 
This difference appeared for patients without cancer history $(P<0.001)$, but not for those with such a history.

\section{Discussion}

This is an observational study that compares the survival of two groups of patients with NSCLC stage IIIB: those treated with chemoradiotherapy versus those treated only with chemotherapy. These two comparable groups were formed exclusively by patients' and/or their families' decision. The same medical team provided the information to patients and their environment. No sociocultural differences were noted between patients of the two groups. Thus, this study is able to provide data that resulted from a pragmatic situation in a chest medicine department.

During the last two decades, different studies have tried to explain the reasons why doctors and patients sometimes do not follow the guidelines. ${ }^{8-10}$ Guidelines are recommendations regarding clinical behavior, and their implementation is a complex process that is influenced by various factors, related both to the characteristics of guidelines themselves and to the social, organizational, economic, and political context or to implementation strategies. Following the guidelines most of all depends on the characteristics linked to the doctor and patient's subjectivity, which can be a real obstacle. Knowledge, attitude, skills, experiences, beliefs, and values play a fundamental role for both physicians and patients. ${ }^{8}$ In addition, treatment guidelines and strategies are based on data from randomized controlled trials and observational clinical studies. These sources drive treatment decisions, yet the data they provide may have limited relevance to the wider population in real-world clinical practice. ${ }^{10}$

The current first line therapeutic option for patients with NSCLC stage IIIB includes platinum-based chemotherapy in combination with a third-generation agent. ${ }^{11}$ Cytotoxic chemotherapy has resulted in improvements in both median overall survival and 1-year survival rates compared with the best supportive care. ${ }^{12}$ Concurrent or sequential radiotherapy is proposed as a complement of chemotherapy. Both add years in terms of survival and palliation of symptoms according to the relative literature. ${ }^{11,13-15}$

The major finding of this study is the confirmation that patients with NSCLC stage IIIB gain in terms of survival from chemoradiotherapy. Patients who received this treatment presented an almost double mean survival compared to those who received only chemotherapy (13.6 versus 7.5 months). Among the group of patients who were treated with chemoradiotherapy, special subgroups gained more from combined therapy. Statistically significant differences were observed in younger patients (aged under 65 years), males, smokers, and patients without cancer history. Using the relative risk model, it was found that the mortality risk for the chemotherapy-treated lung cancer patients who did not receive radiotherapy was independent of the histological subtype of their tumor.

Although, more than $50 \%$ of NSCLC patients are diagnosed over the age of 65 years and approximately one-third of all patients are over the age of 70 years. Elderly patients are often undertreated. Furthermore, patients older than 70 years are under-represented in clinical trials. ${ }^{16}$ Elderly patients are expected to tolerate chemotherapy poorly compared to their younger counterpart, because of the progressive reduction of organ function and comorbidities related to age. ${ }^{17}$ Retrospective analyses suggest that the efficacy of platinumbased chemotherapy is similar in older and younger patients, with increased but acceptable toxicity for elderly patients. Therefore, the outcomes in the elderly mirror results observed in younger patients, although toxicity is generally greater. ${ }^{18}$

Males and smokers are overrepresented in our cohort; therefore no conclusion can be drawn regarding corresponding findings. Patients with cancer history seem to be affected by more aggressive tumors, which result in less responsiveness to combination therapy. ${ }^{19}$ Nevertheless, in a recent study, patients with NSCLC and a previous malignancy did not show a significantly different survival rate than NSCLC patients without history of malignancy. ${ }^{20}$

The lack of a radiotherapy department in our hospital renders impossible the application of concurrent chemoradiotherapy, and therefore leads to the application of the sequential module. The benefits and risks of this option were clearly explained to the patients and/or their families and they were free to make their own decision. Many patients or their families believed that radiotherapy could cause more adverse events than any gain in the quality of life and survival. The majority were discouraged by the need to change hospital and medical staff. These subjective attitudes provoked inequalities in the offer of the proposed treatment. In our study, the need for displacement from one hospital to another was the major complaint of the patients.

Selection biases may as well interfere into the patients' attitudes vis-à-vis the combined treatment (willingness to be treated, social support, socioeconomic status). The presence of a radiotherapy department in our hospital would have alleviated the inequality in the offered treatment. In any case, we had the opportunity to assess the role of the patients' willingness in the application of proposed guidelines for a disease with poor prognosis, as is the case for inoperable lung cancer. 


\section{Conclusion}

The lack of a radiotherapy department in a hospital providing chemotherapy impedes the application of current guidelines advocating the sequential chemoradiotherapy. When recommended radiotherapy after six chemo cycles, half of the patients are unwilling to be displaced and do not follow the recommendations. This has an expected impact on patients' survival.

\section{Disclosure}

No conflicts of interest were declared in relation to this paper.

\section{References}

1. Alberts WM. Introduction: diagnosis and management of lung cancer: ACCP evidence-based clinical practice guidelines (2nd edition). Chest. 2007;132:20-22.

2. Jemal A, Siegel R, Ward E, et al. Cancer statistics 2007. CA Cancer J Clin. 2007;57:43-66.

3. Elias A. Chemotherapy and radiotherapy for regionally advanced nonsmall cell lung cancer. Chest. 1993;103:362-366.

4. Pritchard R, Anthony S. Chemotherapy plus radiotherapy compared with radiotherapy alone in the treatment of locally advanced, unresectable, non-small-cell lung cancer. Cancer. 1995;76:593-601.

5. Rowell NP, O'Rourke NP. Concurrent chemoradiotherapy in non-small cell lung cancer. Cochrane Database Syst Rev. 2004;4:CD002140.

6. Rosenthal DI, Pistenmaa DA, Glatstein E. A review of neoadjuvant chemotherapy for head and neck cancer: partially shrunken tumors may be both leaner and meaner. Int J Radiat Oncol Biol Phys. 1994;28: 315-320.

7. Union Internationale Contre le Cancer. TNM Classification of Malignant Tumours. 6th ed. New York, NY: Wiley-Liss; 2002:272.
8. Baiardini I, Braido F, Bonini M, Compalati E, Canonica GW. Why doctors and patients mot follow guidelines? Curr Opin Allergy Clin Immunol. 2009;9:228-233.

9. Antunes MJ. Guidelines in real life. Why are they not always enforced? Eur J Cardiothorac Surg. 2008;34:935-936.

10. Anderson P, Benford M, Harris N, Karavali M, Piercy J. Real-world physician and patient behaviour across countries: Disease-Specific Programmes - a means to understand. Curr Med Res Opin. 2008 Sep 29. [Epub ahead of print].

11. Pfister DG, Johnson DH, Azzoli CG, et al. American Society of Clinical Oncology treatment of unresectable non-small cell lung cancer guideline: update 2003. J Clin Oncol. 2004;22:330-353.

12. Hanna N, Shepherd FA, Fossella FV, et al. Randomized phase III trial of pemetrexed versus docetaxel in patients with non-small cell lung cancer previously treated with chemotherapy. J Clin Oncol. 2004;22: 1589-1597.

13. Socinski MA, Crowell R, Hensing TE, et al. Treatment of non-small cell lung cancer, stage IV: ACCP evidence-based clinical practice guidelines (2nd edition). Chest. 2007;132:277-289.

14. Ettinger DS, Bepler G, Bueno R, et al. Non-small cell lung cancer: clinical practice guidelines in oncology. J Natl Comp Canc Netw. 2006;4: $548-582$.

15. Reboul FL. Radiotherapy and chemotherapy in locally advanced nonsmall cell lung cancer: preclinical and early clinical data. Hematol Oncol Clin North Am. 2004;18:41-53.

16. Langer CJ. Elderly patients with lung cancer: biases and evidence. Curr Treat Options Oncol. 2002;3:85-102.

17. Rossi A, Gridelli C. Chemotherapy of advanced non-small cell lung cancer in elderly patients. Ann Oncol. 2006;17:58-60.

18. Vamvakas L, Saloustros E, Karampeazis A, Georgoulias V. Advanced non-small-cell lung cancer in the elderly. Clin Lung Cancer. 2009;10: $158-167$.

19. Cassidy A, Balsan J, Vesin A, et al. Cancer diagnosis in first-degree relatives and non-small cell lung cancer risk: results from a multi-centre case-control study in Europe. Eur J Cancer. 2009;45(17):3047-3053.

20. Quadrelli S, Lyons G, Colt H, Chimondeguy D, Silva C. Lung cancer as a second primary malignancy: increasing prevalence and its influence on survival. Ann Surg Oncol. 2009;16:1033-1038.
Cancer Management and Research

\section{Publish your work in this journal}

Cancer Management and Research is an international, peer-reviewed open access journal focusing on cancer research and the optimal use of preventative and integrated treatment interventions to achieve improved outcomes, enhanced survival and quality of life for the cancer patient The journal welcomes original research, clinical \& epidemiological

\section{Dovepress}

studies, reviews \& evaluations, guidelines, expert opinion \& commentary, case reports \& extended reports. The manuscript management system is completely online and includes a very quick and fair peerreview system, which is all easy to use. Visit http://www.dovepress.com/ testimonials.php to read real quotes from published authors. 